\title{
EXTREME ASYMMETRIC DIFFRACTION FOR HIGH FLUX AND COHERENT EXPERIMENTS
}

\author{
Kakuno, E. M. ${ }^{1}{ }^{*}$; Mazzaro, I. ${ }^{2}$; Kellerman, G. ${ }^{2}$; Hönnicke, M. G. ${ }^{3}$; Cusatis, C. ${ }^{2}$ \\ ${ }^{1}$ Universidade Federal do Pampa, Bage, RS, Brazil \\ ${ }^{2}$ Universidade Federal do Parana, DF-LORXI, Curitiba, PR, Brazil \\ ${ }^{3}$ Universidade Federal da Integração Latino-Americana, Foz do Iguaçu, PR, Brazil \\ *edson.kakuno@unipampa.edu.br
}

A device to concentrate a hard X-ray beams $(13.7 \mathrm{keV})$, based on extreme asymmetric diffraction condition in a perfect $\mathrm{Si}$ crystal, is presented. A 75 um thick $\mathrm{Si}$ (100) slab was used in order to select the asymmetric $\mathrm{Si}(606)$ diffraction plane. The thin Si (100) crystal was positioned at the normal incidence respect to the incoming X-ray beam. Then, the diffracted beam was detected at the extreme grazing exit, i.e., at the edge of the thin crystal. The experiment was performed at the XRD2 beamline in the Brazilian Synchrotron (LNLS). The beam was collimated by a Rh coated silicon mirror. Downtream, a Si(111) double crystal monochromator (DCM) was tuned at 13,697.5 $\mathrm{eV}$. The angular full width of half maximum (FWHM) of the diffracted beam [Si(606) reflection], measured by angular scanning of a $50 \mathrm{um}$ slit at $0.28 \mathrm{~m}$ from the sample, was determined to be $580 \mu \mathrm{rad}$. Such device opens up new possibilities in X-ray imaging since it can be used as a secondary high flux coherent source for phase contrast X-ray imaging. It is worth noticing that ultra-thin $\mathrm{Si}$ crystals ( $<5 \mu \mathrm{m}$ thick) are now available and can be also used in such a device in order to procuce high flux microfocus X-ray sources. 